\title{
ON THE IRREDUCIBILITY OF NONUNITARY INDUCED REPRESENTATIONS OF CERTAIN SEMIDIRECT PRODUCTS
}

\author{
BY \\ ERNEST THIELEKER( $\left.{ }^{1}\right)$
}

\begin{abstract}
Let $G$ be a connected Lie group which is a semidirect product of a compact subgroup $K$ and a normal solvable subgroup $S$. Let $\Lambda$ be a character of $S$, and let $M_{\Lambda}$ be the stabilizer of $\Lambda$ in $K$. Let $[H, \Lambda \mu]$ be a finite-dimensional irreducible representation of the subgroup $S M_{\mathrm{A}}$ on the complex vector space $H$. In this paper we consider the induced representations of $G$ on various Banach spaces, and study their topological irreducibility. The basic method used consists in studying the irreducibility of the Lie algebra representations which arise on the linear subspaces of $K$-finite vectors. The latter question then can be reduced to the problem of determining when certain modules over certain commutative algebras are irreducible. The method discussed in this paper leads to two theorems giving sufficient conditions on the character $\Lambda$ that the induced representations be topologically irreducible. The question of infinitesimal equivalence of various induced representations is also discussed.
\end{abstract}

Introduction. Let $G$ be a connected Lie group which is a semidirect product of a compact subgroup $K$ and a normal solvable subgroup $S$. Let $\Lambda$ be a character of $S$, that is, a one-dimensional complex representation of $S$. Let $M_{\Lambda}$ be the stabilizer of this character; that is $M_{\Lambda}$ is the subgroup defined by the expression

$$
M_{\Lambda}=\left\{a \in K: \Lambda\left(a s a^{-1}\right)=\Lambda(s), s \in S\right\} .
$$

Then $S M_{\Lambda}$ is a closed subgroup of $G$. Let $[H, \mu]$ be a finite-dimensional irreducible representation of $M_{\Lambda}$, and let $\Lambda \mu$ be the representation of the subgroup $S M_{\Lambda}$ defined by the formula $\Lambda \mu(s m) x=\Lambda(s) \mu(m) x, x \in H, s \in S$, and $m \in M_{\Lambda}$. Then we assert that the representation of the group $G$ induced by the representation $\Lambda \mu$ of the subgroup $S M_{\Lambda}$ is topologically irreducible, under some general conditions on the character $\Lambda$. (A review of the basic concepts needed for this discussion will appear below.) In case the representation $\Lambda \mu$ is unitary and $S$ is abelian, the result mentioned above leads to known results, which follow from Mackey's theory of unitary induced representations as applied to semidirect products. Of course, in the unitary case, similar results are also known for much more general semidirect product groups than the ones we consider here. The method that we

Received by the editors November 24, 1970 .

AMS 1970 subject classifications. Primary 22E45; Secondary 22D30.

$\left.{ }^{(}\right)$Work begun under the auspices of the U.S. A.E.C. while at Argonne National Laboratory, and completed at the University of South Florida.

Copyright (C) 1972, American Mathematical Society 
describe here works for unitary as well as nonunitary representations, and consists of examining the Lie algebra modules of $K$-finite vectors which arise from the induced representation. We point out that the basic argument used here also works, in conjunction with additional machinery, in the case where $G$ is a real semisimple group. A discussion of the latter case will be taken up in a subsequent paper.

The principal methods used consist in reducing the problem of irreducibility of a Lie algebra to the question of uniform density of a certain commutative algebra of functions. (See Lemma 3.) The latter question is then examined by more-or-less classical methods: in particular, the theory of unitary representations of compact groups, and the Stone-Weierstrass theorem. The principal application of our methods is to the proof of Theorems 1 and 4. We also consider the question of equivalence of the induced representations. The chief result in this direction is Theorem 2.

The author wishes to thank Professor J. M. G. Fell for example (a) (§9). Consideration of this example made it possible to modify the author's previously incorrect formulation of the main result of this paper.

1. Review of some basic concepts. Let $G$ be a connected Lie group. We do not assume at this stage of the discussion that $G$ is a semidirect product. Let $K$ be a compact subgroup of $G$. Let $\left[H_{\pi}, \pi\right]$ be a continuous representation of the group $G$ on a Banach space $H_{\pi}\left({ }^{2}\right)$. Such a representation is said to be $K$-finite if the restriction of $\pi$ to $K$ splits into a direct sum of $K$-irreducible representations such that each class of irreducible representations of $K$ occurs with finite multiplicity. More precisely, the space $H_{\pi}$ contains a dense linear subspace $d H_{\pi}$ which is invariant under the restriction of $\pi$ to $K$, and which splits as a $K$-module into a direct sum of finite-dimensional $K$-isotypic subspaces. A $K$-isotypic subspace is one on which $K$ acts as a multiple of an irreducible representation. Let $\Omega(K)$ denote the set of equivalence classes of irreducible $K$-modules. Thus, assuming that $H_{\pi}$ is $K$-finite, we denote by $\omega H_{\pi}$ the finite-dimensional subspace of $H_{\pi}$ belonging to the class $[\omega]$. Then the linear space $d H_{\pi}$ consists of finite linear combinations of elements in the subspaces $\omega H_{\pi},[\omega] \in \Omega(K)$.

Assume that $\left[H_{\pi}, \pi\right]$ is a $K$-finite representation of $G$. The following facts follow easily from the analytic vector theorem of Nelson, and from results of HarishChandra [3]. (See [8] for a more detailed review in the present context.)

(a) The linear subspace $d H_{\pi}$ consists of analytic vectors; in particular, each element of this subspace is infinitely differentiable. Hence, it makes sense to apply the operators $d \pi(Z)$ to these elements, defined, for each element $Z$ in the Lie algebra $\left({ }^{3}\right) \boldsymbol{G}$ of the group $G$, by the formula

$$
d \pi(Z)=d \pi(\exp t Z) /\left.d t\right|_{t=0}
$$

$\left({ }^{2}\right)$ As a matter of notation we shall indicate a representation $g \rightarrow \pi(g)$ in a linear space $H_{\pi}$ by the symbol $\left[H_{\pi}, \pi\right]$. Similarly the representations of a Lie algebra shall be denoted by $\left[d H_{\pi}, d \pi\right]$.

$\left({ }^{3}\right)$ In general, if $L$ is a Lie group, we denote by $L$ its Lie algebra. 
It is known that the linear space $d H_{\pi}$ is invariant under the representation $Z \rightarrow d \pi(Z)$ of the Lie algebra $G$, and since the vectors in $d H_{\pi}$ are infinitely differentiable, one may extend this representation to a representation of the universal enveloping algebra $U(\boldsymbol{G})$ of the Lie algebra $\boldsymbol{G}$. We also denote the latter representation by the symbol $\left[d H_{\pi}, d \pi\right]$.

(b) The group representation $\left[H_{\pi}, \pi\right]$ is topologically irreducible if and only if the Lie algebra representation $[d H, d \pi]$ is algebraically irreducible. More generally, we have a one-to-one correspondence between closed invariant subspaces $K_{\pi}$ of the subspace $H_{\pi}$ and submodules $\left[d K_{\pi}, d \pi\right]$ of the module $\left[d H_{\pi}, d \pi\right]$, such that $K_{\pi}$ is the closure of the linear space $d K_{\pi}$.

Two $K$-finite representations $\left[H_{\pi}, \pi\right]$ and $\left[H_{\pi}^{\prime}, \pi^{\prime}\right]$ are said to be infinitesimally equivalent if and only if their corresponding $G$-modules $\left[d H_{\pi}, d \pi\right]$ and $\left[d H_{\pi}^{\prime}, d \pi^{\prime}\right]$ are algebraically equivalent. This is a true equivalence relation, and is the same as the relation of Naimark equivalence. (See Fell [2, p. 296].)

Now let us assume that the group $G$ is a semidirect product of a normal solvable subgroup $S$ and the compact subgroup $K$. This condition implies of course that $G$ is homeomorphic to the topological product $S \times K$, and there exists a homomorphism of $K$ into Aut $(S): k \rightarrow \tau(k)$, such that $k s k^{-1}=\tau(k) s$ for all $k \in K$, and $s \in S$. We note that it follows from the first condition that $G$ is actually analytically isomorphic to the product manifold $S \times K$. The proof of the last fact is the same as the proof in Helgason [4, p. 235].

Now let $\Lambda$ be a character of the subgroup $S$; hence $M_{\Lambda}$ is closed in $K$. Hence $S M_{\Lambda}$ is a closed subset of $G$. The fact that $S M_{\Lambda}$ is actually a subgroup follows from the following observation for $m, m^{\prime} \in M$, and $s, s^{\prime} \in S:(s m)^{-1}=\tau\left(m^{-1}\right) s^{-1} m^{-1}$ $\in S M_{\Lambda} ; s m s^{\prime} m^{\prime}=s \tau(m) s^{\prime} m m^{\prime} \in S M_{\Lambda}$.

We now review two definitions of induced representations in this setting. These two definitions turn out to be infinitesimally equivalent. Let $[H, \mu]$ be the given finite-dimensional irreducible representation of $M_{\Lambda}$. We equip the space $H$ with an inner product $($,$) and with the resulting finite-dimensional Hilbert space$ structure. Let $L_{\Lambda \mu}^{2}(G, H)$ denote the usual Hilbert space of equivalence classes of functions $f$ which satisfy the condition

$$
f(p g)=\Lambda \mu(p) f(g), \quad p \in S M_{\Lambda}, \quad \text { and } g \in G,
$$

and such that the following Haar integral over $K$ exists:

$$
\|f\|_{2}=\langle f, f\rangle=\int_{K}(f(k), f(k)) d k .
$$

Similarly we denote by the symbol $C_{\Lambda \mu}(G, H)$ the space of continuous functions from $G$ to $H$ which satisfy the condition (1). We equip this space with the Banach norm

$$
\|f\|_{\infty}=\sup _{k \in K}(f(k), f(k))^{1 / 2}
$$


It is clear that both the spaces $L_{\Lambda \mu}^{2}(G, H)$ and $C_{\Lambda \mu}(G, H)$ are invariant under right translations by elements of $G$. Let us denote this representation by $g \rightarrow R(g)$. It is well known and easy to verify that this action of $G$ is continuous on each of these two Banach spaces. We call the representations $\left[L_{\Lambda \mu}^{2}(G, H), R\right]$ and $\left[C_{\Lambda \mu}(G, H), R\right]$ the induced and continuous induced representations of $G$ respectively. By relation (1), the functions in either of these Banach spaces are uniquely determined by their restrictions to $K$. Let $L_{\mu}^{2}(K, H)$ and $C_{\mu}(K, H)$ denote the spaces of such restrictions of elements in $L_{\Lambda \mu}^{2}(G, H)$ and $C_{\Lambda \mu}(G, H)$ respectively. Now the space $C_{\mu}(K, H)$ is dense in the space $L_{\mu}^{2}(K, H)$ and the action of $K$ on the latter space by right translations is just the induced representation of $K$, induced by the representation $\mu$ of the subgroup $M_{\Lambda}$. In particular, we deduce from this fact that each class $[\omega] \in \Omega(K)$ occurs with a multiplicity $(\omega: \mu)$, by the Frobenius reciprocity theorem applied to compact groups, where $(\omega: \mu)$ is the multiplicity of $\mu$ in any representation $\omega$ of the class $[\omega]$. (See Weil $[9$, p. 82$]$.) Hence, the class $[\omega]$ occurs with this multiplicity in the restriction of $R$ to $K$ in each of the spaces $L_{\Lambda \mu}^{2}(G, H)$ and $C_{\Lambda \mu}(G, H)$. Moreover we have $\omega L_{\Lambda \mu}^{2}(G, H)=\omega C_{\Lambda \mu}(G, H)$ for all classes $[\omega] \in \Omega(K)$. Hence, for each representation $\Lambda \mu$ of the subgroup $S M_{\Lambda}$, the spaces of $K$-finite vectors $d L_{\Lambda \mu}^{2}(G, H)$ and $d C_{\Lambda \mu}(G, H)$ are dense in their respective spaces, and are in fact equal. The density of these spaces is an easy consequence of the Peter-Weyl theorem. (See Weil in the above reference.) Thus both the induced and continuous induced representations are $K$-finite and infinitesimally equivalent to each other.

2. Statement of the main result. In the notation set up in the last section, $\left[d C_{\Lambda \mu}(G, H), d R\right]$ is the representation of the Lie algebra $\boldsymbol{G}$ acting on the space of $K$-finite vectors of $L_{\Lambda \mu}^{2}(G, H)$ as well as of the space $C_{\Lambda \mu}(G, H)$. The statement of the main theorem (Theorem 1) depends on a condition on the character $\Lambda$. In order to state this condition we need some preliminary remarks.

Since $S$ is a normal subgroup of $G$ it follows that $S$, the Lie algebra of $S$, is invariant under the adjoint representation of $G$. Hence, in particular, it is invariant under the restriction of the adjoint representation to $K$. Let us denote the latter representation by $\rho$. In other words $[S, \rho]$ is the representation defined by the formula $\rho(k) Z=\operatorname{Ad}(k) Z$, for $k \in K$, and $Z \in S$. Let $\langle,>$ be an inner product on the vector space $S$. Since $K$ is compact, we may choose the inner product in such a manner that the representation $\rho$ is orthogonal with respect to the finitedimensional Euclidean space structure which this inner product induces on the space $\boldsymbol{S}$. The last observation is the result of a standard argument. Let $\boldsymbol{S}_{\boldsymbol{C}}$ denote the complexification of $\boldsymbol{S}$. Then the inner product $\langle$,$\rangle may be extended to an Her-$ mitian inner product on $S_{C}$ by the definition $\left\langle x+I y, x^{\prime}+I y^{\prime}\right\rangle=\left\langle x, x^{\prime}\right\rangle+\left\langle y, x^{\prime}\right\rangle$ $+I\left(\left\langle y, x^{\prime}\right\rangle-\left\langle x, y^{\prime}\right\rangle\right)$, for $x, y, x^{\prime}, y^{\prime} \in \boldsymbol{S}$. Then $\left[\boldsymbol{S}_{C}, \rho\right]$ is unitary with respect to this structure.

We note that there exists a linear form $\lambda$ on $S_{C}$, defined by the equation $\lambda(Z)$ $=d \Lambda(\exp t Z) /\left.d t\right|_{t=0}$ for all $Z \in S$. It follows that $\lambda$ is a one-dimensional representa- 
tion of the Lie algebra $S$. It follows from this fact that $\lambda$ vanishes on the subalgebra $[\boldsymbol{S}, \boldsymbol{S}]$; however, we will not use the latter property explicitly. Let us identify the vector space $S_{C}$, the complexification of $S$, with its vector dual by means of the form $\langle$,$\rangle . If \lambda \in S_{C}$ we may write $\lambda$ in the form $\lambda_{R}+I \lambda_{I}$, with $\lambda_{R}, \lambda_{I} \in S$. We define the complex conjugate $\bar{\lambda}$ of the element $\lambda$ by the formula $\bar{\lambda}=\lambda_{R}-I \lambda_{I}$.

If $\Lambda$ is a character on the group $S$, with corresponding infinitesimal character $\lambda$, then $\bar{\Lambda}$ shall denote the character corresponding to the complex conjugate from $\bar{\lambda}$. If $\Lambda$ is a character, we shall say that $\Lambda$ is cyclic if the corresponding infinitesimal character $\lambda$ is a cyclic vector in $S_{C}$ for the representation $\rho$.

THEOREM 1. Assume that the character $\bar{\Lambda}$ is cyclic in the sense described above. Then for each irreducible finite-dimensional representation $[H, \mu]$ of $M_{\Lambda}$, the subspace $d C_{\Lambda \mu}(G, H)$ is an irreducible $G$-module under the action $d R$. Moreover, the induced representation and the continuous induced representation of $G$ induced by the representation $\Lambda \mu$ of $S M_{\Lambda}$ are both topologically irreducible.

RemarK. In case $\Lambda$ is the trivial character, $M_{\Lambda}=K$. The spaces $C_{\Lambda \mu}(G, H)$, $d C_{\Lambda \mu}(G, H)$, etc. reduce to the space of constant functions. In this case the $G$ module is simply the trivial extension of the module $[H, \mu]$ to $G: R(s k)=\mu(k)$, $s \in S, k \in K$. The conclusion of the theorem follows trivially in this case.

By what has been said in $\S 1$, the second statement of the theorem follows from the first statement, which concerns Lie algebra modules.

3. On the structure of the module $\left[d C_{\Lambda \mu}(G, H), d R\right]$. Let $C_{\Lambda}^{\infty}(G, H)$ denote the vector space of infinitely differentiable functions from $G$ to $H$ which satisfy the condition $f(s g)=\Lambda(s) f(g)$, for $f \in C_{\Lambda}^{\infty}(G, H), s \in S$, and $g \in G$. Since every function in $d C_{\Lambda \mu}(G, H)$ is infinitely differentiable (even analytic) it follows from equation (1) that we have the inclusion $C_{\Lambda}^{\infty}(G, H) \supset d C_{\Lambda \mu}(G, H)$. We are going to compute the action of $d R(S)$ on the space $C_{\Lambda}^{\infty}(G, H)$.

Lemma 1. Let $Y$ be an element of $S$, and let $f \in C_{\Lambda}^{\infty}(G, H)$. Then $d R(Y) f$ is the function in $C_{\Lambda}^{\infty}(G, H)$ given by $k \rightarrow\langle\rho(k) Y, \lambda\rangle f(k), k \in K$. (Note that each function in $C_{\Lambda}^{\infty}(G, H)$ is uniquely determined by its restriction to $K$ because of the relation $f(s k)=\Lambda(s) f(k), s \in S, k \in K$.)

Proof. It is obvious that the space $C_{\Lambda}^{\infty}(G, H)$ is invariant under right translations, $R(g)$, by elements of $G$. Hence, for $Z \in S$, and $f \in C_{\Lambda}^{\infty}(G, H)$, the function $d R(Z) f$ $=d R(\exp Z) f /\left.d t\right|_{t=0}$ lies in $C_{\Lambda}^{\infty}(G, H)$. Moreover we have, with $k \in K$,

$$
\begin{aligned}
d R(Z) f(k) & =\left.\frac{d}{d t} f(k \exp t Z)\right|_{t=0}=\left.\frac{d}{d t} f(\tau(k) \exp t Z k)\right|_{t=0}=\left.\frac{d}{d t} f(\exp t \rho(k) Z k)\right|_{t=0} \\
& =\left.\frac{d}{d t} \Lambda(\exp t \rho(k) Z) f(k)\right|_{t=0}=\lambda(\rho(k) Z) f(k)=\langle\rho(k) Z, \lambda\rangle f(k) .
\end{aligned}
$$

The fourth expression follows from the definition of the space $C_{\Lambda}^{\infty}(G, H)$, while the second from the last expression follows from the definition of the form $\lambda$. Q.E.D. 
For any element $0 \neq \lambda \in S_{C}$, let $\mathscr{S}(\lambda)$ denote the algebra generated over the complex numbers by 1 and the functions $k \rightarrow\langle\rho(k) Y, \lambda\rangle$, under pointwise multiplication. Then the space $C_{\Lambda}^{\infty}(G, H)$ is an $\mathscr{S}(\lambda)$-module with the following definition of multiplication: $(\phi f)(s k)=\phi(k) f(s k), s \in S, k \in K$. In other words, the multiplication is pointwise provided the functions in $\mathscr{S}(\lambda)$ are regarded as functions on $G$ by the trivial extension $\phi(s k)=\phi(k), s \in S, k \in K$.

By a repeated application of the above lemma, we have the following formula for any monomial $Y_{1} \cdots Y_{n}$ in the universal enveloping algebra $U(S)$ :

$$
d R\left(Y_{1} \cdots Y_{n}\right) f(k)=d R\left(Y_{1}\right) \cdots d R\left(Y_{n}\right) f(k)=\left\langle\rho(k) Y_{1}, \lambda\right\rangle \cdots\left\langle\rho(k) Y_{n}, \lambda\right\rangle f(k) .
$$

By the theorem of Birkhoff and Witt, the algebra $U(\boldsymbol{S})$ is spanned linearly by these monomials. We note also that the complexification of $U(\boldsymbol{S})$ is just $U\left(\boldsymbol{S}_{C}\right)$. Thus, we have proved the following lemma:

\section{LEMma 2. For any $f \in C_{\Lambda}^{\infty}(G, H)$, we have $d R\left(U\left(S_{C}\right)\right) f=\mathscr{S}(\lambda) f$.}

Let us indicate now what result is still needed to prove irreducibility of the $R(K)$ module of $K$-finite vectors. In order to prove that $d C_{\Lambda \mu}(G, H)$ is irreducible as a $d R(U(G))$-module, it must be shown that every nonzero element $f \in d C_{\Lambda \mu}(G, H)$ is cyclic for this module structure; that is, we must show that we have the following equality of spaces: $d C_{\Lambda \mu}(G, H)=d R(U(G)) f$. However, we may view the algebras $U(\boldsymbol{S})$ and $U(\boldsymbol{K})$ as subalgebras of the algebra $U(\boldsymbol{G})$ generated by the sets $\{1, \boldsymbol{S}\}$ and $\{1, K\}$ respectively. By standard methods, it can be shown that we have the equality $U(\boldsymbol{G})=U(\boldsymbol{S}) U(\boldsymbol{K})$. (See for example, Harish-Chandra [3, p. 193].) Hence for any $f \in d C_{\Lambda \mu}(G, H)$ we have the equality $d R(U(\boldsymbol{G})) f=d R(U(\boldsymbol{S})) d R(U(\boldsymbol{K})) f$. However, $f$ is a $K$-finite element. Hence, the subspace $\mathscr{F}$ defined by $\mathscr{F}=d R(U(K)) f$ is a finite-dimensional $K$-invariant subspace of the space $d C_{\Lambda \mu}(G, H)$. Hence, it is sufficient to show that the space $d C_{\Lambda \mu}(G, H)$ is generated as a $d R(U(\boldsymbol{S}))$-module over any finite-dimensional $K$-invariant subspace $\mathscr{F}$. However, by Lemma 2 , the last statement is equivalent to saying that the space $d C_{\Lambda \mu}(G, H)$ is generated as an $\mathscr{S}(\lambda)$-module over any finite-dimensional $K$-invariant subspace $\mathscr{F}$. The next section will be devoted to a proof of the last statement.

4. The basic reduction. In the last section we reduced the problem of studying the irreducibility of the Lie algebra module $\left[d C_{\Lambda \mu}(G, H), d R\right]$ to the problem of studying the $\mathscr{S}(\lambda)$-module structure of the space of $K$-finite vectors $d C_{\Lambda \mu}(G, H)$. In this section we reduce the latter problem to the special case where $\mu$ is the trivial representation of the subgroup $M_{\Lambda}$, and $H$ is just the one-dimensional space $C$.

Let $C_{\mu}(K, H)$ and $L_{\mu}^{2}(K, H)$ denote the spaces introduced at the end of $\S 1$. It is clear that these spaces may be defined, without explicit reference to the character $\Lambda$, as follows. The space $C_{\mu}(K, H)$ is the space of continuous functions from $K$ to $H$ which satisfy the condition

$$
f(m k)=\mu(m) f(k),
$$


for $m \in M_{\Lambda}$, and $k \in K$. Then $L_{\mu}^{2}(K, H)$ may be defined as the completion of the space $C_{\mu}(K, H)$ in the Hilbert space structure defined by the Haar integral in equation (2). For each class $[\omega] \in \Omega(K)$ let $C_{\mu}(K, H)$ denote $R(K)$-isotypic subspace of $L_{\mu}^{2}(K, H)$. That is, the finite-dimensional subspace of elements which transform like $[\omega]$ under right translations by elements of $K$. Let $d C_{\mu}(K, H)$ denote the linear span of such $R(K)$-isotypic subspaces.

For the special case in which $\mu$ is the identity representation of the subgroup $M_{\Lambda}$, and $H=C$, we denote the space $C_{\mu}(K, H)$ by the symbol $C_{0}^{\lambda}(K)$. In other words, $C_{0}^{\lambda}(K)$ consists of all continuous functions from $K$ to $C$ which satisfy the relationship $f(m k)=f(k)$, for $m \in M_{\Lambda}$, and $k \in K$. It should be noted that the space $C_{0}^{\lambda}(K)$ may also be interpreted as the space $C\left(M_{\Lambda} \mid K\right)$, consisting of all continuous complexvalued functions on the space of right cosets $M_{\Lambda} \mid K$. In fact if $f$ is such a function and if $\tau$ is the natural continuous projection of $K \rightarrow$ into $M_{\Lambda} \backslash K$ then $f \circ \tau \in C_{0}^{\lambda}(K)$. Conversely if $f \in C_{0}^{\lambda}(K)$ then $f$ is obviously constant on each coset in $M_{\Lambda} \backslash K$, and may then be viewed as a continuous function on cosets. We identify the spaces $C_{0}^{\lambda}(K)$ and $C\left(M_{\Lambda} \mid K\right)$. It is clear that $\mathscr{S}(\lambda)$ is a linear subspace of $C_{0}^{\lambda}(K)=C\left(M_{\Lambda} \mid K\right)$. Finally let $L_{0}^{2}(K)$ or $L^{2}\left(M_{\Lambda} \mid K\right)$ denote $L^{2}$-completion of the space $C_{0}^{\lambda}(K)$ or $C\left(M_{\Lambda} \mid K\right)$ in the Hilbert space norm defined by equation (1).

For the purposes of the following discussion, we may ignore the fact that $S$ is a Lie algebra, and consider the following setup. $\left[\boldsymbol{S}_{\boldsymbol{C}}, \rho\right]$ is a finite-dimensional representation of $K$ on a complex vector space $S_{C}$. Let $\lambda$ be a nonzero element of this space, and let $M_{\Lambda}$ be the closed subgroup of $K$ which fixes this element in the representation $\rho$. As before, $\langle$,$\rangle is an inner product on S_{C}$ which makes the representation $\rho$ unitary, and $\mathscr{S}(\lambda)$ is the algebra generated by 1 and functions of the form $k \rightarrow\langle\rho(k) Z, \lambda\rangle, Z \in S_{C}$. The representation $[H, \mu]$ is an irreducible representation of the subgroup $M_{\Lambda}$, and $\left[L_{\mu}^{2}(K, H), R\right]$ is the representation of $K$ which this representation of $M_{\Lambda}$ induces. Then the basic result of this section is the following:

LEMMA 3. Let $[H, \mu]$ be an irreducible finite-dimensional representation of $M_{\Lambda}$. Then the following statements are equivalent:

(i) $\mathscr{S}(\lambda)$ is dense in $L^{2}\left(M_{\Lambda} \mid K\right)$.

(ii) Let $\mathscr{F}$ be a nontrivial $R(K)$-invariant subspace of $C_{\mu}(K, H)$. Then $\mathscr{S}(\lambda) \mathscr{F}$ is dense in $L_{\mu}^{2}(K, H)$.

(iii) Let $\mathscr{F}$ be as in (ii) then $\mathscr{S}(\lambda) \mathscr{F}=d C_{\mu}(K, H)$.

Proof. (i) $\rightarrow$ (ii). Assume $\mathscr{S}(\lambda)$ is dense in $L^{2}\left(M_{\Lambda} \mid K\right)$. We will first show that the space $\mathscr{S}(\lambda) \mathscr{F}$ is dense in $L_{\mu}^{2}(K, H)$. Let $D$ denote the orthogonal complement of $\mathscr{S}(\lambda) \mathscr{F}$ in the Hilbert space $L_{\mu}^{2}(K, H)$. If the space $\mathscr{S}(\lambda) \mathscr{F}$ were not dense, $D$ would contain a nonzero element $f$, and we would have the vanishing of the Haar integral: $\int_{K}(f(k), g(k)) d k=0$, for all elements $g \in \mathscr{S}(\lambda) \mathscr{F}$. We recall that, in the last equation, $($,$) is the inner product on H$. Let $F$ be a nonzero element of the finite-dimensional $K$-invariant subspace $\mathscr{F}$. Then for all $\phi \in \mathscr{S}(\lambda)$ we have $(f(k), \phi(k) F(k))$ 
$=\phi(k)(f(k), F(k))$, where the "bar" denotes complex conjugation. Hence the vanishing of the last integral implies the vanishing of the integral

$$
\int \phi(k)(f(k), F(k)) d k=0 \quad \text { for all } \phi \in \mathscr{S}(\lambda) \text {. }
$$

However, the function $k \rightarrow(f(k), F(k))$ clearly lies in the space $L_{0}^{2}(K)$. Hence, this function must vanish identically, by (i). Now, since $\mathscr{F}$ is $K$-invariant, under the action of right translations, and since $F$ was an arbitrary element of this subspace in the above argument, it follows that for all $k, k^{\prime} \in K$ we have $\left(f(k), F\left(k k^{\prime}\right)\right)$ $=\left(f(k), R\left(k^{\prime}\right) F(k)\right)=0$. Or, in other words, $\left(f(k), F\left(k^{\prime}\right)\right)=0$ for all $k, k^{\prime} \in K$. By taking a right translate of $F$, if necessary, we may assume that $F(e) \neq 0$. Because of the irreducibility of the representation $[H, \mu]$ of the subgroup $M_{\Lambda}$, we find that the set of elements $\mu(m) F(e)$, for $m \in M_{\Lambda}$, spans $H$. However, by relation $\left(1^{\prime}\right)$, this last set of elements is just the set $\left\{F(m): m \in M_{\Lambda}\right\}$. Hence for all $k \in K$, the value of the function $f$ at $k$ is orthogonal to $H$; hence it is zero, since it must lie in $H$. Thus we have shown that $f=0$. This conclusion contradicts the assumption that $D \neq 0$. Hence $\mathscr{S}(\lambda) \mathscr{F}$ is dense in $L_{\mu}^{2}(K, H)$.

(ii) $\rightarrow$ (iii). We first point out that every element in $\mathscr{S}(\lambda)$ is $K$-finite. Hence, we have the inclusion $\mathscr{S}(\lambda) \mathscr{F} \subset d C_{\mu}(K, H)$. Let $R(\omega)$ denote the orthogonal projection on $L_{\mu}^{2}(K, H)$ projecting on the subspace of $R(K)$-isotypic components corresponding to $[\omega] \in \Omega(k)$. Because this projection is finite dimensional, and because the space $\mathscr{S}(\lambda) \mathscr{F}$ is dense, we have $R(\omega) \mathscr{S}(\lambda) \mathscr{F}=R(\omega) L_{\mu}^{2}(K, H)$. However, the latter subspaces span $d C_{\mu}(K, H)$, by definition of the latter. Hence we have $\mathscr{S}(\lambda) \mathscr{F}$ $=d C_{\mu}(K, H)$.

(iii) $\rightarrow$ (i). Take $\mathscr{F}$ to be the subspace of constant functions and take $[H, \mu]$ to be the identity representation of $M_{\Lambda}$. Q.E.D.

5. On the density of the space $\mathscr{S}(\lambda)$. The lemma of the last section shows that the question of irreducibility of the $G$-module $\left[d C_{\Lambda \mu}(G), d R\right]$ may be reduced to the problem of deciding when the space $\mathscr{S}(\lambda)$ is dense in $L^{2}\left(M_{\Lambda} \backslash K\right)$. The main lemma of this section (Lemma 5) is the key lemma of this argument and is based mainly on the Stone-Weierstrass theorem. It is related to an argument used by Hochschild and Mostow [5, p. 515]. Before presenting this argument we need a few preliminary remarks.

Let $\Lambda$ be a nontrivial character on $S$, and let $\lambda$ be the corresponding linear form in $S_{C}$. (See §2.) As in $\S 2$, we may write $\lambda=\lambda_{R}+I \lambda_{I}$, with $\lambda_{R}, \lambda_{I} \in S$. Then we let $\Lambda_{R}$ and $\Lambda_{I}$ be the characters on $S$ corresponding to the linear forms $\lambda_{R}$ and $\lambda_{I}$ respectively. We assert the following.

LEMMA 4. We have the equality of subgroups. $M_{\Lambda}=M_{\Lambda_{R}} \cap M_{\Lambda_{I}}$.

Proof. Since $M_{\Lambda}=\{k \in K: \rho(k) \lambda=\lambda\}, \quad M_{\Lambda_{R}}=\left\{k \in K: \rho(k) \lambda_{R}=\lambda_{R}\right\}$, and $M_{\Lambda_{I}}$ $=\left\{k \in K: \rho(k) \lambda_{I}=\lambda_{I}\right\}$, it is clear that $M_{\Lambda_{R}} \cap M_{\Lambda_{I}} \subset M_{\Lambda}$. Let $k \in M_{\Lambda}$ then $\rho(k)\left(\lambda_{R}+I \lambda_{I}\right)=\lambda_{R}+I \lambda_{I}$. However, $\rho(k) \lambda_{R}$, and $\rho(k) \lambda_{I}$ are "real" vectors for all 
$k \in K$. That is, these vectors lie in $S$. Hence, by taking real and imaginary parts of the last equation, we have $\rho(k) \lambda_{R}=\lambda_{R}$ and $\rho(k) \lambda_{I}=\lambda_{I}$. That is $k \in M_{\Lambda_{R}} \cap M_{\Lambda_{I}}$. Q.E.D.

As usual, we denote the operation of conjugation on complex-valued functions by $\phi \rightarrow \phi$. Thus $\phi$ is defined by

$$
\phi(k)=\overline{\phi(k)} \text { for } k \in K .
$$

We now state the main result of the section.

LEMMA 5. The following conditions are equivalent:

(i) $\mathscr{S}(\bar{\lambda}) \subset \mathscr{S}(\lambda)$,

(ii) $\mathscr{S}(\lambda)$ is closed under complex conjugation,

(iii) $\mathscr{S}(\lambda)$ contains a nontrivial finite-dimensional real-linear subspace consisting of real-valued continuous functions, and which distinguishes points on $M_{\Lambda} \backslash K$,

(iv) $\mathscr{S}(\lambda)$ is dense in $C\left(M_{\Lambda} \mid K\right)=C_{0}^{\lambda}(K)$ (i.e., uniformly dense),

(v) $\mathscr{S}(\lambda)$ is dense in $L^{2}\left(M_{\Lambda} \mid K\right)$,

(vi) $\mathscr{S}(\lambda)=d C\left(M_{\lambda} \mid K\right)=d C\left(M_{\Lambda} \backslash K\right)$; i.e., $\mathscr{P}(\lambda)$ consists of all the $R(K)$-finite vectors in $d C\left(M_{\lambda} \mid K\right)$.

Proof. (i) $\rightarrow$ (ii). For each $X \in \boldsymbol{S}_{\boldsymbol{C}}$, and $\mu \in \boldsymbol{S}_{\boldsymbol{C}}$, let $k \rightarrow \phi(k ; X, \mu)$ denote the function on $K$ defined by the equation $\phi(k ; X, \mu)=\langle\rho(k) X, \mu\rangle$. We note in general, by a straightforward computation, that $\langle X, Y\rangle^{-}=\langle\bar{X}, \bar{Y}\rangle$ for all $X, Y \in \boldsymbol{S}_{C}$. Now, since the representation $k \rightarrow \rho(k)$ fixes the real subspaces $S$ and $I S$, we have for all $k \in K$,

$$
\phi(k ; X, \mu)=\overline{\phi(k ; X, \mu)}=\langle\overline{\rho(k) X, \mu\rangle}=\langle\rho(\overline{k) X} \bar{\mu}\rangle=\langle\rho(k) \bar{X}, \bar{\mu}\rangle=\phi(k ; \bar{X}, \bar{\mu}) .
$$

Now every element in $\mathscr{S}(\lambda)$ is a polynomial of functions of the form $\phi(k ; X, \lambda)$, $X \in S_{C}$. The conjugate of such a function must then be a polynomial of functions of the form $\phi(k ; \bar{X}, \bar{\lambda}), \bar{X} \in \boldsymbol{S}_{C}$, and thus lies in $\mathscr{S}(\bar{\lambda}) \subset \mathscr{S}(\lambda)$.

(ii) $\rightarrow$ (iii). Assume $\mathscr{P}(\lambda)$ is closed under complex conjugation. Let $\lambda_{R}$ and $\lambda_{I}$ be elements of $S$ such that $\lambda=\lambda_{I}+I \lambda_{R}$. Let $\mathscr{S}_{1 R}$ be the real-linear space given by the real span:

$$
\mathscr{S}_{1 R}=\operatorname{span}_{R}\left\{\phi\left(\cdot ; X, \lambda_{R}\right), \phi\left(\cdot ; Y, \lambda_{I}\right): X, Y \in S\right\} .
$$

It is clear that every function in this space is real, and is obviously finite dimensional. We assert that this subspace distinguishes points on $M_{\Lambda} \backslash K$. Let $k_{1} \in K$, and $k_{2} \in K$ such that $\phi\left(k_{1} ; X, \mu\right)=\phi\left(k_{2} ; X, \mu\right)$ for all $X \in S$, and all $\mu \in \operatorname{span}_{R}\left\{\lambda_{R}, \lambda_{I}\right\}$. Then we have

$$
0=\left\langle\rho\left(k_{1}\right) X, \mu\right\rangle-\left\langle\rho\left(k_{2}\right) X, \mu\right\rangle=\left\langle X, \rho\left(k_{1}^{-1}\right) \mu-\rho\left(k_{2}^{-1}\right) \mu\right\rangle
$$

for all $X \in S$. This statement implies that $\rho\left(k_{1}^{-1}\right) \mu=\rho\left(k_{2}^{-1}\right) \mu$ or that $\rho\left(k_{2} k_{1}^{-1}\right) \mu=\mu$. In particular we have $\rho\left(k_{2} k_{1}^{-1}\right) \lambda_{R}=\lambda_{R}$, and $\rho\left(k_{2} k_{1}^{-1}\right) \lambda_{I}=\lambda_{I}$, so that $k_{2} k_{1}^{-1} \in M_{\Lambda_{I}}$ $\cap M_{\Lambda_{R}}$. However, by Lemma 4, the latter intersection is equal to $M_{\Lambda}$. Thus $k_{1}$ and $k_{2}$ lie in the same coset modulo $M_{\Lambda}$. 
(iii) $\rightarrow$ (iv). Let $\mathscr{R}$ be a finite-dimensional real subspace of $\mathscr{S}(\lambda)$ consisting of real-valued functions which distinguishes points on $M_{\Lambda} \mid K$. Let $\mathscr{R}_{C}$ be the complexification of this subspace. Then $\mathscr{R}_{C}$ consists of functions of the form $f+I g, f, g \in \mathscr{R}$, and is thus closed under complex conjugation. Let $A$ be the pointwise algebra generated by $C$ and $\mathscr{R}$. Then we have the inclusion $\mathscr{R} \subset \mathscr{R}_{C} \subset A \subset \mathscr{S}(\lambda)$. The algebra $A$ is closed under conjugation. Hence by the Stone-Weierstrass theorem, $A$ is uniformly dense in $C\left(M_{\Lambda} \mid K\right)$. Hence $\mathscr{P}(\lambda)$ is uniformly dense in $C\left(M_{\Lambda} \mid K\right)$.

(iv) $\rightarrow(\mathrm{v})$. Assume $\mathscr{S}(\lambda)$ is dense in $C\left(M_{\Lambda} \mid K\right)$. Since $M_{\Lambda} \mid K$ is compact, uniform convergence on $M_{\Lambda} \mid K$ implies $L^{2}$-convergence. Since $C\left(M_{\Lambda} \mid K\right)$ is dense in $L^{2}\left(M_{\Lambda} \mid K\right)$ it follows that $\mathscr{S}(\lambda)$ is dense in $L^{2}\left(M_{\Lambda} \mid K\right)$.

$(\mathrm{v}) \rightarrow(\mathrm{vi})$. Assume $\mathscr{S}(\lambda)$ is dense in $L^{2}\left(M_{\Lambda} \mid K\right)$. In any case we have $\mathscr{S}(\lambda)$ $\subset d C\left(M_{\Lambda} \mid K\right)$. Now, if $\mathscr{S}(\lambda) \neq d C\left(M_{\Lambda} \mid K\right)$ there must exist class $[\omega] \in \Omega(K)$ such that the finite-dimensional subspace $R(\omega) \mathscr{S}(\lambda)$ is a proper subspace of the finitedimensional subspace $R(\omega) C\left(M_{\Lambda} \mid K\right)$. Let $A$ be the orthogonal complement of $R(\omega) \mathscr{S}(\lambda)$ in $R(\omega) C\left(M_{\Lambda} \mid K\right)$. By the Schur orthogonality relations $A$ then lies in the orthogonal complement of all the subspaces, $R\left(\omega^{\prime}\right) \mathscr{S}(\lambda)$, for $\left[\omega^{\prime}\right] \in \Omega(K)$. Hence $A$ lies in the orthogonal complement of $\mathscr{S}(\lambda)$, a contradiction.

(vi) $\rightarrow$ (i). Assume that $\mathscr{S}(\lambda)=d C\left(M_{\Lambda} \mid K\right)$. It is clear that $\mathscr{S}(\bar{\lambda})$ consists of $K$-finite vectors. Moreover by Lemma 4 we have $M_{\bar{\Lambda}}=M_{\Lambda_{R}} \cap M_{\Lambda_{l}}=M_{\Lambda}$. Hence $\mathscr{S}(\bar{\lambda}) \subset d C\left(M_{\Lambda} \mid K\right)=\mathscr{S}(\lambda)$. Q.E.D.

6. Proof of the main theorem. Assume that the character $\bar{\Lambda}$ is cyclic in the sense described in $\S 2$. In terms of the infinitesimal characters, we recall that this condition implies that the function $k \rightarrow \phi(k ; \bar{\lambda}, \lambda)=\langle\rho(k) \bar{\lambda}, \lambda\rangle$ does not vanish. We note first of all that this function is real. In fact since the representation $k \rightarrow \rho(k)$ fixes the real subspaces $\boldsymbol{S}$ and $I S$, it follows that, for all $k \in K$,

$$
\langle\overline{\rho(k) \bar{\lambda}, \lambda}\rangle=\langle\overline{\rho(k) \bar{\lambda}}, \bar{\lambda}\rangle=\langle\rho(k) \lambda, \bar{\lambda}\rangle=\left\langle\rho(k)^{-1} \bar{\lambda}, \lambda\right\rangle .
$$

We assert that the latter function is actually equal to the function $k \rightarrow\langle\rho(k) \bar{\lambda}, \lambda\rangle$ $=\phi(k ; \lambda, \lambda)$. It will follow from this assertion that this function is real. Let us postpone the proof of this assertion and see first how it leads to a proof of the theorem:

Let $\mathscr{R}$ be the real span of the right translates, by elements of $K$, of the function $\phi(\cdot ; \bar{\lambda}, \lambda)$. By the last assertion, this function is real. Hence $\mathscr{R}$ consists of realvalued functions. Moreover, since $\phi(\cdot ; \bar{\lambda}, \lambda)$ is $K$-finite, $\mathscr{R}$ is finite dimensional. We wish to apply Lemma 5 to show that $\mathscr{S}(\lambda)$ is dense in $L^{2}\left(M_{\Lambda} \mid K\right)$. We must show that $\mathscr{R}$ distinguishes points on $M_{\Lambda} \backslash K$. In other words, we must show that if $f\left(k_{1}\right)=f\left(k_{2}\right)$ for all $f \in R$ then $k_{2} k_{1}^{-1} \in M_{\Lambda}$. In other words, we assume that for any finite set $g_{1}, \ldots, g_{n} \in K$ and complex numbers $\lambda_{1}, \ldots, \lambda_{n}$ we have

$$
\sum_{1}^{n} \lambda_{i} R\left(g_{i}\right) \phi\left(k_{1} ; \bar{\lambda}, \lambda\right)=\sum_{1}^{n} \lambda_{i} R\left(g_{i}\right) \phi\left(k_{2} ; \lambda, \lambda\right)
$$


or

$$
\sum_{1}^{n} \lambda_{i} R\left(g_{i}\right)\left[\left\langle\bar{\lambda}, \rho\left(k_{1}\right)^{-1} \lambda\right\rangle-\left\langle\bar{\lambda}, \rho\left(k_{2}^{-1}\right) \lambda\right\rangle\right]=0
$$

or

$$
\left\langle\sum_{1}^{n} \lambda_{i} \rho\left(g_{i}\right) \bar{\lambda}, \rho\left(k_{1}\right)^{-1} \lambda-\rho\left(k_{2}^{-1}\right) \lambda\right\rangle=0 .
$$

However, $\bar{\lambda}$ is assumed to be cyclic for the representation $\left[\boldsymbol{S}_{C}, \rho\right]$. Hence $\rho\left(k_{1}\right)^{-1} \lambda$ $=\rho\left(k_{2}^{-1}\right) \lambda$ or $k_{2} k_{1}^{-1} \in M_{\Lambda}$. Thus, it follows that $\mathscr{S}(\lambda)$ is dense by Lemma 5 .

The proof of the theorem now follows easily. Let $S_{C}$ now stand for the complexification of the Lie algebra $S$, again. Every function in the space $d C_{\Lambda \mu}(G, H)$ is uniquely determined by its restriction to $K$ in $d C_{\mu}(K, H)$, because of the relation $f(s k)=\Lambda(s) f(k)$, for $s \in S$, and $k \in K$. Now, Lemma 3 shows that the space $d C_{\Lambda \mu}(G, H)$ is generated, as an $\mathscr{S}(\lambda)$-module over any finite-dimensional $K$-invariant subspace, in the sense described in the third section. Hence, it follows from that discussion that $\left[d C_{\Lambda \mu}(G, H), d R\right]$ is an irreducible representation of $\boldsymbol{G}$. Finally, it follows immediately from the density of $d C_{\mu}(K, H)$ in $L_{\mu}^{2}(K, H)$ that the space $d C_{\Lambda \mu}(G, H)$ is dense in the space $L_{\Lambda \mu}^{2}(G, H)$. Similarly, from the uniform density of the space $d C_{\mu}(K, H)$ in $C_{\mu}(K, H)$, it follows that the subspace $d C_{\Lambda \mu}(G, H)$ is uniformly dense in $C_{\Lambda \mu}(G, H)$. Hence, the last statement of the theorem follows. Q.E.D. Theorem.

We now turn to the remaining gap in the proof of Theorem 1, namely, the following assertion.

Lemma 6. Assume that the function $k \rightarrow \phi(k ; \bar{\lambda}, \lambda)$ does not vanish. Then $\phi(k ; \bar{\lambda}, \lambda)$ $=\phi\left(k^{-1} ; \bar{\lambda}, \lambda\right)$ for any $\lambda \in \boldsymbol{S}_{C}$.

Proof. Let $n=$ dimension $S$, and let $\left\{e_{1}, \ldots, e_{n}\right\}$ be an orthonormal basis. Then this basis is also an orthonormal basis in $S_{C}$. For each $k \in K, \rho(k)$ is a real orthogonal matrix on $S$. Hence by a standard result in linear algebra, there exists a real orthogonal matrix $A$ such that the matrix $A \rho(k) A^{-1}$ has the form

$$
A \rho(k) A^{-1}=\text { diagonal }\left[\left(\begin{array}{cc}
\cos \theta_{1} & \sin \theta_{1} \\
-\sin \theta_{1} & \cos \theta_{1}
\end{array}\right) \cdots\left(\begin{array}{cc}
\cos \theta_{p} & \sin \theta_{p} \\
-\sin \theta_{p} & \cos \theta_{p}
\end{array}\right), 1, \ldots, 1\right]
$$

where the number of 1's along the diagonal is $n-2 p$; moreover the angles $\theta_{i}$, $i=1, \ldots, p$, are real. Let the matrix shown be denoted by $d\left(\theta_{1}, \theta_{2}, \ldots, \theta_{p}\right)$. Then we have

$$
A \rho\left(k^{-1}\right) A^{-1}=\left[A \rho(k) A^{-1}\right]^{-1}=d\left(-\theta_{1},-\theta_{2}, \ldots,-\theta_{p}\right) .
$$

Finally we note that since $A$ is orthogonal on $S_{C}$ we have $A \bar{\lambda}=(A \lambda)^{-}$. Let $\mu_{1}, \ldots, \mu_{n}$ be the complex coefficients such that $A \lambda=\sum \mu_{i} e_{i}$. Then we have

$$
\begin{aligned}
\langle\rho(k) \bar{\lambda}, \lambda\rangle & =\left\langle A^{-1} d\left(\theta_{1} \cdots \theta_{p}\right) A \bar{\lambda}, \lambda\right\rangle=\left\langle d\left(\theta_{1} \cdots \theta_{p}\right) A \bar{\lambda}, A \lambda\right\rangle \\
& =\left[\left(\bar{\mu}_{1}^{2}+\bar{\mu}_{2}^{2}\right) \cos \theta_{1}+\cdots+\left(\bar{\mu}_{2 p-1}^{2}+\bar{\mu}_{2 p}^{2}\right) \cos \theta_{p}\right] \\
& =\left\langle d\left(-\theta_{1} \cdots-\theta_{p}\right) A \bar{\lambda}, A \lambda\right\rangle=\left\langle\rho\left(k^{-1}\right) \bar{\lambda}, \lambda\right\rangle \text { Q.E.D. }
\end{aligned}
$$


7. Some results on the equivalence of induced representations. We would like to determine when two induced representations of the type discussed are infinitesimally equivalent. In other words, we would like to determine when the corresponding Lie algebra representations on $K$-finite vectors are algebraically equivalent. Unfortunately, we must be content with slightly less information. However, the results of this section provide complete information in the unitary case. (See the next section.)

For each $x \in K$, we define the map $\Lambda \rightarrow \Lambda^{x}$ on the characters (one-dimensional representations of $S$ ) to be the dual of the automorphism $\tau(x): \Lambda^{x}(s)=\Lambda(\tau(x) s)$ $=\Lambda\left(x s x^{-1}\right)$, for all $s \in S$. It is a straightforward matter to verify that the subgroups $M_{\Lambda^{x}}$, and $M_{\Lambda}$ are conjugate: $M_{\Lambda^{x}}=x^{-1} M_{\Lambda} x$. Let $\mu^{x}$ denote the representation of the subgroup $M_{\Lambda^{x}}$ defined by $\mu^{x}\left(x^{-1} m x\right)=\mu(m)$, for $m \in M$. We then have the following theorem.

THEOREM 2. Assume that the continuous induced representation $\left[C_{\Lambda \mu}(G, H), R\right]$ is topologically irreducible. Then the continuous induced representations of $G:\left[C_{\Lambda \mu}(G, H), R\right]$ and $\left[C_{\Lambda^{\prime} \mu^{\prime}}(G, H), R\right]$ are equivalent (in the Banach space sense) if and only if the following two conditions hold:

(1) The characters $\Lambda^{\prime}$ and $\Lambda$ lie on the same orbit under $k \rightarrow \tau(k)$; in other words, there exists an $x \in K$ such that $\Lambda^{\prime}=\Lambda^{x}$.

(2) Let $x$ be the element of the last sentence. Then the representation $\left[H^{\prime}, \mu^{\prime}\right]$ is a representation of $M_{\Lambda^{x}}$ equivalent to the representation $\left[H, \mu^{x}\right]$.

REMARK. It is clear that the theorem implies that the last two conditions are sufficient that representations induced by $\Lambda^{\prime} \mu^{\prime}$ and $\Lambda \mu$ lie in the same infinitesimal equivalence class. However, in general it is not clear that these two conditions are necessary. (It is possible that there may exist an infinitesimal intertwining map which is not continuous in the uniform topology.) However, if it is known that $\left[C_{\Lambda \mu}(G, H), R\right]$ is infinitesimally equivalent to a unitary representation, then $C_{\Lambda^{\prime} \mu^{\prime}}\left(G, H^{\prime}\right)$ must also be infinitesimally equivalent to a unitary representation. It follows then from a result of Harish-Chandra (see [3, Theorem 8]) that the representations $\left[L_{\Lambda \mu}^{2}(G, H), R\right]$ and $\left[L_{\Lambda^{\prime} \mu^{\prime}}^{2}\left(G, H^{\prime}\right), R\right]$ are unitarily equivalent. Such an equivalence must be continuous in the uniform topology on the subspaces of continuous functions. Hence, it follows from Theorem 2 that the conditions listed there are necessary as well as sufficient in the unitary case. A weaker set of necessary conditions is given in the next theorem.

Proof of Theorem 2. First assume that conditions (1) and (2) hold. Let $\mathscr{T}$ be an intertwining map from the representation $\left[H, \mu^{x}\right]$ to the representation $\left[H^{\prime}, \mu^{\prime}\right]$; this map exists by (2). Then a straightforward calculation shows that the map $T: f \rightarrow T f$, defined by $T f(g)=\mathscr{T} L(x) f(g)=\mathscr{T} f(x g)$, maps the space $C_{\Lambda \mu}(G, H)$ into the space $C_{\Lambda^{\prime} \mu^{\prime}}\left(G, H^{\prime}\right)$ and intertwines the representation $d R(G)$. The map $T$ is obviously continuous. Hence by the irreducibility of these two representations, this map is bijective. 
The proof in the opposite direction is a little more involved. Let $T$ be an intertwining map from the Banach space $C_{\Lambda^{\prime} \mu^{\prime}}\left(G, H^{\prime}\right)$ onto the Banach space $C_{\Lambda \mu}(G, H)$. First, we note that if $\Lambda^{\prime}$ is the trivial character, then $M_{\Lambda^{\prime}}=K$. The space $C_{\Lambda^{\prime} \mu^{\prime}}\left(G, H^{\prime}\right)$ then simply coincides with the constant functions to $H^{\prime}$. Moreover, it follows from Lemma 1 that the Lie algebra $\boldsymbol{S}$ acts trivially on this space, and by the same lemma, $C_{\Lambda \mu}(G, H)$ is clearly infinite dimensional unless $\Lambda=1$, the trivial character. Hence $M_{\Lambda}=K$ also, and condition (2) follows.

Now, let us assume that both $\Lambda$ and $\Lambda^{\prime}$ are nontrivial characters on $S$. As in $\S 2$, we define $\lambda$ and $\lambda^{\prime}$ to be the one-dimensional Lie algebra representations of $S$ corresponding to the characters $\Lambda$ and $\Lambda^{\prime}$ respectively.

For each $Z \in S$ let $k \rightarrow \phi(k ; Z, \lambda)$ and $k \rightarrow \phi\left(k ; Z, \lambda^{\prime}\right)$ denote the functions $\langle\rho(\cdot) ; Z, \lambda\rangle$ and $\left\langle\rho(\cdot) Z, \lambda^{\prime}\right\rangle$ respectively. Then the functions $\phi(\cdot ; Z, \lambda)$ and $\phi\left(\cdot ; Z, \lambda^{\prime}\right)$ together with the constant functions generate the algebras $\mathscr{S}(\lambda)$ and $\mathscr{S}\left(\lambda^{\prime}\right)$ respectively. We will show that there exists a nontrivial homomorphism $\lambda$ of the algebra $\mathscr{S}\left(\lambda^{\prime}\right)$ into the complex numbers, and that this homomorphism has the following properties: (a) $\lambda(1)=1$, and $\lambda\left(\phi\left(\cdot ; Z, \lambda^{\prime}\right)=\lambda(Z)\right.$ for all $Z \in S$. (b) The homomorphism $\lambda$ is continuous in the uniform topology on $\mathscr{S}\left(\lambda^{\prime}\right)$. Indeed, let $\delta$ denote the linear map from $C_{\Lambda \mu}(G, H)$ to the space $H$ defined by evaluation at the identity: $\delta(f)=f(e)$. Then, by Lemma 1 and the intertwining property of the map $T$, we have for all $Z, W \in S$, and all $f \in d C_{\Lambda^{\prime} \mu^{\prime}}\left(G, H^{\prime}\right)$, the following calculations.

$$
\delta\left(T \phi\left(\cdot ; Z, \lambda^{\prime}\right) f\right)=\delta(T d R(Z) f)=\delta(d R(Z) T f)=\delta(\phi(\cdot ; Z, \lambda) T f)=\lambda(Z) \delta(T f) .
$$

Hence,

$$
\delta\left(T \phi\left(\cdot ; Z, \lambda^{\prime}\right) \phi\left(\cdot ; W, \lambda^{\prime}\right) f\right)=\lambda(Z) \delta\left(T \phi\left(\cdot ; W, \lambda^{\prime}\right) f\right)=\lambda(Z) \lambda(W) \delta(T f) .
$$

Now pick an $f \in d C_{\Lambda^{\prime} \mu^{\prime}}\left(G, H^{\prime}\right)$ and an element $v$ in the dual of $H$ such that the number $v(\delta T f)$ is not zero. Then define $\lambda$ as the linear map from $\mathscr{S}\left(\lambda^{\prime}\right)$ to $C: \lambda(\phi)$ $=v(\delta T \phi f) / v(\delta T f)$, for all $\phi \in \mathscr{S}\left(\lambda^{\prime}\right)$. The first line of calculation shows that property (a) is satisfied, and the second line shows that $\lambda$ has the homomorphism property on the generators of the algebra $\mathscr{S}\left(\lambda^{\prime}\right)$; hence, it is a homomorphism. Finally the continuity of $\lambda$ follows from the continuity of the intertwining map $T$.

We recall, that by Lemma 3 and the assumption of irreducibility, the uniform closure of the algebra $\mathscr{S}\left(\lambda^{\prime}\right)$ is the space $C_{0}^{\lambda^{\prime}}(K)$ of all continuous complex-valued functions on $K$ which satisfy the condition $f(m k)=f(k)$, for $m \in M_{\Lambda^{\prime}}$, and $k \in K$. As pointed out before, every function with the last stated property may be viewed as a function on the homogeneous space of left cosets: $M_{\Lambda^{\prime}} \mid K$, and every function that is continuous with respect to the quotient topology on this homogeneous space may be viewed as a function in $C_{0}^{\lambda^{\prime}}(K)$. Since the coset space $M_{\Lambda^{\prime}} \backslash K$ is a compact Hausdorff space, it follows from a well-known result in the theory of commutative Banach algebras that the set of all maximal ideals in the algebra $C_{0}^{\lambda^{\prime}}(K)$ is just the set of kernels of the evaluation maps. Hence, there exists a coset $[y] \in M_{\Lambda^{\prime}} \mid K$ such that $\lambda(\phi)=\phi(y)$, for all $y \in[y]$ and all $\phi \in C_{0}^{\lambda^{\prime}}(K)$. 
Now, let us fix an element $x^{-1}$ in the coset [y], and apply the homomorphism $\lambda$ to the functions $\phi\left(\cdot ; Z, \lambda^{\prime}\right)$, for each $Z \in S$. We obtain

$$
\lambda^{\prime}\left(\rho\left(x^{-1}\right) Z\right)=\lambda\left(\phi\left(\cdot ; Z, \lambda^{\prime}\right)\right)=\lambda(Z),
$$

for all $Z \in S$. Hence, $\Lambda^{\prime}\left(\tau\left(x^{-1}\right) s\right)=\Lambda(s)$, for all $s \in S$; that is $\Lambda^{\prime}=\Lambda^{x}$. This proves condition (1) of the theorem.

In order to prove condition (2), we note that the $K$-modules $\left[L_{\mu}^{2}(K, H), R\right]$ and $\left[L_{\mu^{\prime}}^{2}\left(K, H^{\prime}\right), R\right]$ are equivalent. Since the subgroups $M_{\Lambda^{x}}$ and $M_{\Lambda}$ are isomorphic, it follows that every irreducible character of $M_{\Lambda^{x}}$ is also an irreducible character of $M_{\Lambda}$. Hence, by the Frobenius reciprocity theorem for compact groups (see Weil $\left[9\right.$, p. 82]) we have $(\omega: \mu)=\left(\omega: \mu^{\prime}\right)$ for $[\omega] \in \Omega(K)$. Hence, for every class function $f$ on $M_{\Lambda}$, we have the following equality of Haar integrals over $M_{\Lambda}: \int f(m) \bar{\chi}_{\mu}(m) d m$ $=\int f(m) \bar{\chi}_{\mu^{\prime}}(m) d m$. Hence, by the Peter-Weyl theorem, $\chi_{\mu}=\chi_{\mu^{\prime}}$ or $\mu^{\prime}$ is equivalent to $\mu^{x}$. Q.E.D.

As we pointed out above, we do not know that conditions (1) and (2) in the above theorem are necessary, although they are clearly sufficient, for infinitesimal equivalence. However, a slight modification of the above argument can yield the following result.

THEOREM 3. Let $n=$ dimension $\left(\boldsymbol{S}_{C}\right)$. By means of the map $k \rightarrow \rho\left(k^{-1}\right) \lambda$, we identify the left coset space $M_{\Lambda} \mid K$ with the orbit in $S_{C}$ through the point $\lambda$. In order that $\left[d C_{\Lambda^{\prime} \mu^{\prime}}\left(G, H^{\prime}\right) d R\right]$ be algebraically equivalent to the $G$-module $\left[d C_{\Lambda \mu}(G, H) d R\right]$ it is necessary that $\lambda$ lie on the Zariski closure of the orbit of $\lambda^{\prime}$, and hence $\lambda^{\prime}$ must lie on the Zariski closure of the orbit of $\lambda$. If $\lambda$ and $\lambda^{\prime}$ lie on the same orbit then condition (2) of the last theorem must hold.

Proof. Let $Z_{1}, \ldots, Z_{n}$ be a basis of $S_{C}$. Then the algebra of polynomial functions generated over $C$ by the functions $\lambda \rightarrow\left\langle Z_{i}, \lambda\right\rangle, i=1, \ldots, n$, is isomorphic to the algebra $C^{n}[x]$ of polynomials in $n$-indeterminants $\left(x_{1}, \ldots, x_{n}\right)$, over $C$. Then obviously, the algebra $\mathscr{S}\left(\lambda^{\prime}\right)$ may be thought of as the algebra of restrictions of the polynomial functions to the orbit $k^{-1} \rightarrow \rho(k) \lambda^{\prime}$. Let $I$ be the kernel of this restriction homomorphism. Let $R(I)$ denote the radical of $I$. Then the Zariski closure is the zero set of $R(I)$.

Now, the proof of this theorem is identical with the proof of the last theorem, with the following exception. The homomorphism $\lambda^{\prime}$ constructed there is not necessarily continuous in the uniform topology on $\mathscr{S}\left(\lambda^{\prime}\right)$. Hence, we cannot characterize this homomorphism in terms of the structure space of $C_{0}^{\lambda^{\prime}}(K)$ as we did there. We only know that there exists a point $[y]$ in the Zariski closure of the orbit of $\lambda^{\prime}$ such that $\lambda(\phi)=\phi(y)$, for all $\phi \in \mathscr{S}\left(\lambda^{\prime}\right)$. Q.E.D. Theorem.

8. Another irreducibility theorem. In this section we present another irreducibility theorem which follows easily from our considerations. For this result we do not assume that the character $\bar{\Lambda}$ is cyclic, but instead, we assume that $\Lambda$ is proportional to a real character. By the latter condition we mean that there exists a 
complex number $c \neq 0$, such that $c \lambda \in S$, where again $\lambda$ is the infinitesimal character in $\boldsymbol{S}_{\boldsymbol{C}}$ corresponding to $\Lambda$. For example all unitary characters are proportional to real characters in this sense, since $I \lambda \in S$, for that case.

THEOREM 4. Assume that $\Lambda$ is proportional to a real character. Then for each irreducible finite-dimensional representation $[H, \mu]$ of $M_{\Lambda}$, the subspace $d C_{\Lambda \mu}(G, H)$ is an irreducible $G$-module under the action $d R$. Moreover, the induced representations and the continuous induced representation of $G$, induced by the representation $\Lambda \mu$ of $S M_{\Lambda}$, are both topologically irreducible.

Proof. The proof of this theorem is similar to the proof of Theorem 1 . The only difference is the construction of the real subspace $\mathscr{R}$, which is simpler in the present situation. In fact let $c$ be a nonzero complex number such that $c \lambda \in S$, and let $\mathscr{R}$ be defined as

$$
\mathscr{R}=\{c \phi(\cdot ; Z, \lambda): Z \in S\} .
$$

Obviously $\mathscr{R}$ is a real-linear subspace of $\mathscr{S}(\lambda)$ of finite dimension. To show that $\mathscr{R}$ distinguishes points on $M_{\Lambda} \mid K$, we assume that for $k_{1}, k_{2} \in K$, we have $c \phi\left(k_{1} ; Z, \lambda\right)$ $=c \phi\left(k_{2} ; Z, \lambda\right)$, for all $Z \in S$. Then $\left\langle Z, \rho\left(k_{1}\right)^{-1} \lambda-\rho\left(k_{2}\right)^{-1} \lambda\right\rangle=0$ for all $Z \in S$, which implies that $\rho\left(k_{1}\right)^{-1} \lambda=\rho\left(k_{2}\right)^{-1} \lambda$ or $k_{2} k_{1}^{-1} \in M_{\Lambda}$. Hence, $k_{1}$ and $k_{2}$ lie in the same coset modulo $M_{\Lambda}$. The proof now is identical to that of Theorem 1. Q.E.D.

9. Some examples. (a) First we present an example, essentially due to Fell, in which both the hypotheses of Theorem 1 and of Theorem 4 fail to be satisfied.

Let $G$ be the real two-dimensional Euclidean group. Then $S$ is the vector addition group of the real two-dimensional vector space, and this underlying space may be identified with the Lie algebra $S$ equipped with trivial Lie multiplication. The group $K$ is the group of rotations in $S$. Let us write $K=\{\theta: 0 \leqq \theta \leqq 2 \pi \equiv 0\}$. Let $\left\{e_{1}, e_{2}\right\}$ be an orthonormal basis of $S$. Let $\Lambda$ be the character on $S$ whose infinitesimal form is $\lambda=e_{1}+I e_{2}$. Then $M_{\Lambda}=\{e\}$, and $\rho(\theta) \bar{\lambda}=\exp (-I \theta)$. Hence $\bar{\Lambda}$ is not cyclic. It is also clear that $\Lambda$ is not proportional to a real vector. We note that in this case we have

and

$$
\mathscr{S}(\lambda)=\operatorname{span}\{\theta \rightarrow \exp \operatorname{Im} \theta: m=0,1,2, \ldots\}
$$

$$
\mathscr{S}(\bar{\lambda})=\operatorname{span}\{\theta \rightarrow \exp -\operatorname{Im} \theta: m=0,1,2, \ldots\} .
$$

So that statement (i) in Lemma 5 does not hold; in fact we actually have $\mathscr{S}(\lambda) \cap \mathscr{S}(\bar{\lambda})=C$. We note the Lie algebra module $\left[d C_{\Lambda}(K), d R\right]$ is not irreducible. In fact, it follows from Lemma 1 that for each integer $M$, the following is an invariant subspace.

$$
\mathscr{S}(\lambda) \exp (\cdot) I M=\operatorname{span}_{C}\{\theta \rightarrow \exp \operatorname{Im} \theta: m=M, M+1, \ldots\} .
$$

Hence the induced representation is not irreducible. We note on the other hand, that if $\lambda \neq 0$ is not an eigenvector of the representation $\theta \rightarrow \rho(\theta)$, then $\lambda$ is a cyclic vector so that Theorem 1 implies irreducibility. 
(b) Let $G$ be the Euclidean group on real $n$-dimensional space with $n \geqq 3$. Then $S$ is the real $n$-dimensional vector space with the operation of vector addition. Again $S$ has the same underlying vector space as $S$ with abelian Lie multiplication. $K$ may be taken as the group of orthogonal transformations on $S$ and $\rho$ is the identity map. For this example $\rho$ remains irreducible in the complexification $\boldsymbol{S}_{\boldsymbol{C}}$. Hence, every nonzero vector in $\boldsymbol{S}_{C}$ is cyclic, so that Theorem 1 applies for any nontrivial character $\Lambda$. We remark that Lemma 3 implies that $\mathscr{S}(\lambda)$ is dense in that case, a result of some independent interest.

We remark that in this example, there are two possible orbit types for the action of $K$ on $S_{C}$. If $\Lambda$ is proportional to a real vector as in the last section, then $M_{\Lambda} \mid K$ is an $(n-1)$-sphere, and if $\Lambda$ is not proportional to a real vector then $M_{\Lambda} \cong S O(n-2)$ $\times S O(2)$, and $M_{\Lambda} \mid K$ is a compact manifold of dimension $n(n-1) / 2$.

(c) Another special case of some interest is the motion group associated with a real connected simple Lie group. This example is obtained as follows. Let $G_{0}$ be a connected simple Lie group and let $K$ be a maximal compact subgroup of $G$. Let $\boldsymbol{P}$ be a Cartan subspace of $G_{0}$ (see for example Helgason [4, Chapter 3]). Then $K$ acts on $\boldsymbol{P}$ by the representation: $\rho(k) Z=\operatorname{Ad}(k) Z, Z \in \boldsymbol{P}$. Equip $\boldsymbol{P}$ with the structure of vector addition. We define the motion group $G$ associated with $G_{0}$ as the semidirect product of $\boldsymbol{P}$ and $K$ with the action $\rho$. It is known (see for example Helgason) that the representation $[\boldsymbol{P}, \rho]$ is an irreducible representation of $K$. Over the complex numbers two things can happen:

(1) $\left[\boldsymbol{P}_{C}, \rho\right]$ is irreducible,

(2) $\left[\boldsymbol{P}_{C}, \rho\right]$ splits into a direct sum $\boldsymbol{P}_{C}=\boldsymbol{P}^{+}+\boldsymbol{P}^{-}$.

This is the case when $G$ is the isometry group of an Hermitian symmetric space.

For case (1) the situation of irreducibility is the same as for case (b) above. For case (2), $K$ has a one-dimensional center, say $Z_{1}=\{\exp \theta z: 0 \leqq \theta \leqq 2 \pi\}, z \in \boldsymbol{Z}$ ( $\boldsymbol{Z}$ is the one-dimensional center of $K$, the Lie algebra of $K$ ). The situation here is a generalization of example (a): If $0 \neq \lambda$ is not an eigenvector of $\rho(Z)$, then $\bar{\lambda}$ is cyclic so that Theorem 1 applies. If $\lambda$ is an eigenvector of $\rho(Z)$ then $\mathscr{S}(\lambda) \neq \mathscr{S}(\bar{\lambda})$ and the induced representation is not irreducible.

10. Some remarks on unitary representations. Now let $\Lambda$ be a one-dimensional unitary representation of $S$. For $\Lambda$ to be unitary, it is both necessary and sufficient that the corresponding linear form $\lambda$ defined on $S$ be purely imaginary. We note that Haar measure on $K$ serves as an invariant measure on the homogeneous space $S \backslash G$ under the natural action of $G$ on cosets (see for example Weil [9, p. 45]). Hence, from the now standard discussion of Mackey (see [6, part I, §1]), it follows that the induced representation $\left[L_{\Lambda \mu}^{2}(G, H), R\right]$ is unitary. As we have pointed out in the remark following the statement of Theorem 2 , that theorem together with Theorem 4 gives complete information for this case.

In this case $S$ is abelian, Theorems 4 and 2 combined is a special case of Theorem 14.1 of Mackey [6]. (Since $K$ is a compact Lie group, it is easily shown that Mackey's 
regularity condition obtains.) Since Mackey's argument requires taking a direct integral over all the classes of unitary representations of $S$, that argument breaks down for general $S$, even though we confine our inducing representations to be one-dimensional representations of $S$. A more recent result of Mackey almost includes our result for the unitary case. (See [7, Theorem 8.1].) In order to apply that theorem, one must assume that $S$ is a type one group, an assumption which is not necessary in our argument. Of course, it should be pointed out that once that assumption is made, Mackey's theorem gives much more information for the unitary case. Finally, we remark that when $M_{\Lambda}=$ identity, our result reduces to a result of Bruhat (see [1, Theoréme 6;2]).

\section{REFERENCES}

1. F. Bruhat, Sur les représentations induites des groupes de Lie, Bull. Soc. Math. France 84 (1956), 97-205. MR 18, 907.

2. J. M. G. Fell, Non-unitary dual spaces of groups, Acta Math. 114 (1965), 267-310. MR 32 \#4210.

3. Harish-Chandra, Representations of a semisimple Lie group on a Banach space. I, Trans. Amer. Math. Soc. 75 (1953), 185-243. MR 15, 100.

4. S. Helgason, Differential geometry and symmetric spaces, Pure and Appl. Math., vol. 12, Academic Press, New York, 1962. MR 26 \#2986.

5. G. Hochschild and G. D. Mostow, Representations and representative functions of Lie groups, Ann. of Math. (2) 66 (1957), 495-542. MR 20 \#5248.

6. G. Mackey, Induced representations of locally compact groups. I, Ann. of Math. (2) 55 (1952), 101-139. MR 13, 434.

7. - Unitary representations of group extensions. I, Acta Math. 99 (1958), 265-311. MR 20 \#4789.

8. E. Thieleker, On some infinite dimensional representations of Lie groups (to appear).

9. A. Weil, L'intégration dans les groupes topologiques et ses applications, 2nd ed., Actualités Sci. Indust., no. 869, Hermann, Paris, 1951. MR 3, 198.

Department of Mathematics, University of South Florida, Tampa, Florida 33620 